\title{
Review Article \\ Secretomes from Mesenchymal Stem Cells against Acute Kidney Injury: Possible Heterogeneity
}

\author{
Kenji Tsuji (D), Shinji Kitamura $(\mathbb{D}$, and Jun Wada $(\mathbb{D}$ \\ Department of Nephrology, Rheumatology, Endocrinology and Metabolism, Okayama University Graduate School of Medicine, \\ Dentistry, and Pharmaceutical Sciences, 2-5-1 Shikata-cho, Okayama 700-8558, Japan \\ Correspondence should be addressed to Shinji Kitamura; kitamura@okayama-u.ac.jp
}

Received 20 August 2018; Revised 10 October 2018; Accepted 24 October 2018; Published 16 December 2018

Guest Editor: Zhaoping Ding

Copyright (C) 2018 Kenji Tsuji et al. This is an open access article distributed under the Creative Commons Attribution License, which permits unrestricted use, distribution, and reproduction in any medium, provided the original work is properly cited.

\begin{abstract}
A kidney has the ability to regenerate itself after a variety of renal injuries. Mesenchymal stem cells (MSCs) have been shown to ameliorate tissue damages during renal injuries and diseases. The regenerations induced by MSCs are primarily mediated by the paracrine release of soluble factors and extracellular vesicles, including exosomes and microvesicles. Extracellular vesicles contain proteins, microRNAs, and mRNAs that are transferred into recipient cells to induce several repair signaling pathways. Over the past few decades, many studies identified trophic factors from MSCs, which attenuate renal injury in a variety of animal acute kidney injury models, including renal ischemia-reperfusion injury and drug-induced renal injury, using microarray and proteomic analysis. Nevertheless, these studies have revealed the heterogeneity of trophic factors from MSCs that depend on the cell origins and different stimuli including hypoxia, inflammatory stimuli, and aging. In this review article, we summarize the secretomes and regenerative mechanisms induced by MSCs and highlight the possible heterogeneity of trophic factors from different types of MSC and different circumstances for renal regeneration.
\end{abstract}

\section{Introduction}

Acute kidney injury (AKI) is a worldwide healthcare problem associated with higher risks of mortality and increased length of hospitalization as well as the risk of chronic kidney disease and end-stage renal failure $[1,2]$. In spite of the recent medical advances, few interventions are available other than supportive modalities, such as renal replacement therapies, against AKI. On the other hand, kidney has the ability to regenerate itself after AKI and some patients recover renal function after AKI [3]. Many researchers have tried to elucidate the mechanisms of renal regeneration. Over the past few decades, mesenchymal stem cell- (MSC-) based therapy represents the remarkable strategy to reconstitute the renal tubular formations and attenuate renal function after AKI.

MSCs are multipotent cells with the ability to differentiate into mesodermal lineages, including bone, muscle, chondrocyte, and adipocyte [4]. MSCs can be established from different tissues, such as bone marrow, adipose tissue, synovial tissue, umbilical cord, and connective tissue [4]. MSCs have shown to ameliorate tissue damages caused by renal injuries and diseases. Initially, researchers focused on the differentiation potential of MSCs against renal injury. Actually, MSCs were able to replace renal tubular cells and acquire an epithelial phenotype after renal injury in a murine renal injury model [5]. Nevertheless, the focus on the regenerative effects of MSCs has shifted into their ability to secrete trophic factors. MSCs secrete varieties of cytokines, chemokines, and growth factors as well as extracellular vesicles (EVs) that contain microRNAs (miRNAs), mRNAs, and proteins. Recent reports suggest that the therapeutic activity of MSCs is mainly mediated by the paracrine effect of secretomes. In the past few decades, many studies have identified these secretomes from MSCs and revealed the therapeutic mechanisms associated with cell proliferation, autophagy, cell apoptosis, tissue fibrosis, and inflammation. Meanwhile, recent reports imply the heterogeneity of secretomes of MSCs isolated from different origins. In addition, some 
reports have revealed that different kinds of stimuli affect the secretomes from MSCs. These differences might result in the different outcomes induced by the treatment with MSCs.

In this review article, we summarize the current knowledge about secretomes from MSCs and the therapeutic effects on renal injury and discuss about the possible heterogeneity caused by the differences of cell origins and stimuli.

\section{MSC-Derived Soluble Protein for Renal Generation}

MSCs have been reported to secrete a number of soluble factors including cytokines, chemokines, and growth factors for tissue regeneration. A number of groups have examined proteomic analysis of MSC secretomes to identify regenerative factors against tissue injury. These factors include angiogenic factors [6-8], chemokines [8-10], cytokines $[6,8,10-13]$, growth factors $[8,10-12]$, and other proteins $[10,14-17]$ (Table 1). In general, these proteins exert many biological functions including cell growth, migration, inflammation, apoptosis, and fibrosis. In fact, under the status of kidney injuries, these factors contribute to renal regeneration through antiapoptosis, anti-inflammation, antifibrosis, matrix remodeling, and increased tubular cell proliferation. In addition, a number of reports demonstrate the paracrine effect of MSCs against renal injury. For example, Rota et al. revealed that human amniotic fluid-derived MSCs attenuate cisplatin-induced renal injury through the secretion of trophic factors, such as IL-6, VEGF, and IGF-1 [18]. Lv et al. demonstrated that MSCs ameliorate diabetic glomerular fibrosis via the secretion of BMP-7 [19]. Taken together, MSCs contribute to renal regeneration through the paracrine effect of soluble proteins from MSCs.

\section{MSC-Derived Extracellular Vesicles: Exosomes and Microvesicles}

Recent studies demonstrated that the secreted membrane vesicles called EVs play essential roles on intercellular communications [20-22]. EVs contain various molecules including proteins, miRNAs, and mRNAs. EVs can be organized into several categories such as exosomes $(30-100 \mathrm{~nm}$ diameter), microvesicles (100-1000 nm diameter), and apoptotic bodies (50-5000 $\mathrm{nm}$ diameter) [23]. Increasing evidences have suggested that the MSC-derived EVs might be one of the major contributors for renal regeneration after AKI. Recent analyses identified proteins, miRNAs, and mRNAs in the EVs from MSCs by proteomic and microarray analysis.

\section{4. mRNAs in MSC-Derived Extracellular Vesicles for Renal Regeneration}

mRNAs from EVs transfer into target cells and act via translation into proteins as well as via competing RNAs to regulate stability, localization, and translational activity of mRNAs in target cells $[24,25]$. Bruno et al., for the first time, demonstrated the therapeutic effect of MSC-derived EVs in glycerol-induced AKI in severe combined immunodeficiency
TABLE 1: Soluble factors from MSCs.

\begin{tabular}{|c|c|}
\hline Cytokines & Chemokines \\
\hline IL- $1 \alpha$ & CCL1 \\
\hline IL- $1 \beta$ & CCL2 \\
\hline IL-2 & CCL5 \\
\hline IL-3 & CCL8 \\
\hline IL-6 & CCL11 \\
\hline IL-7 & CCL15 \\
\hline IL-10 & CCL16 \\
\hline IL-11 & CCL18 \\
\hline IL-12 & CCL22 \\
\hline IL-13 & CCL23 \\
\hline IL-16 & CCL24 \\
\hline IFN- $\gamma$ & CCL26 \\
\hline TNF- $\alpha$ & CXCL1 \\
\hline LIF & CXCL2 \\
\hline TGF- $\beta$ & CXCL3 \\
\hline MIF & CXCL5 \\
\hline OSM & CXCL6 \\
\hline G-CSF & CXCL8 \\
\hline M-CSF & CXCL11 \\
\hline GM-CSF & CXCL12 \\
\hline FLT3LG & CXCL13 \\
\hline SCF & CX3CL1 \\
\hline Thrombopoietin & XCL1 \\
\hline \multicolumn{2}{|l|}{ TSG-6 } \\
\hline & Other factors \\
\hline Angiogenic factors & CXCR3 \\
\hline Angiogenin & PGE2 \\
\hline Angiopoietin & PAI-1 \\
\hline \multirow[t]{2}{*}{ VEGF } & MMP1 \\
\hline & MMP3 \\
\hline Growth factors & MMP9 \\
\hline HGF & MMP10 \\
\hline EGF & MMP13 \\
\hline IGF-1 & TIMP-1 \\
\hline FGF-2 & TIMP-2 \\
\hline FGF-4 & TIMP-3 \\
\hline FGF-7 & TIMP-4 \\
\hline FGF-9 & Leptin \\
\hline BMP-7 & IGFBP-1 \\
\hline BDGF & IGFBP-2 \\
\hline GDNF & IGFBP-3 \\
\hline NGF & IGFBP-4 \\
\hline PIGF & Adiponectin \\
\hline \multirow[t]{2}{*}{ PDGF } & Adrenomedullin \\
\hline & Osteoprotegerin \\
\hline
\end{tabular}

IL: interleukin; IFN: interferon; TNF: tumor necrosis factor; LIF: leukemia inhibitory factor; TGF: transforming growth factor; MIF: macrophage migration inhibitory factor; OSM: oncostatin M; G-CSF: granulocyte colony-stimulating factor; M-CSF: macrophage colony-stimulating factor; GM-CSF: granulocyte macrophage colony-stimulating factor; FLT3LG: Fms-related tyrosine kinase 3 ligand; SCF: stem cell factor; TSG-6: TNFstimulated gene 6; VEGF: vascular endothelial growth factors; HGF: hepatocyte growth factor; EGF: epidermal growth factor; IGF: insulin-like growth factor; FGF: fibroblast growth factor; BMP: bone morphogenetic protein; BDNF: brain-derived neurotrophic factor; GDNF: glial cell-derived neurotrophic factor; NGF: nerve growth factor; PIGF: placenta growth factor; PDGF: platelet-derived growth factor; CCL: C-C motif chemokine ligand; CXCL: C-X-C motif chemokine ligand; CX3CL: C-X3-C motif chemokine ligand; XCL: X-C motif chemokine ligand; CXCR: C-X-C motif chemokine receptor; PGE2; prostaglandin E2; PAI: plasminogen activator inhibitor; MMP: matric metalloproteinase; TIMP: tissue inhibitor of metalloproteinase; IGFBP: insulin-like growth factor-binding protein. 
(SCID) mice [26]. Using reverse transcription- (RT-) PCR arrays, they showed that human bone marrow MSCderived EVs contain mRNAs involved in transcription (e.g., CLOCK, IRF6, and LHX6), immune regulation (e.g., CRLF1, $I L 1 R N$, and MT1X), cell cycle regulation (e.g., SENP2, $R B L 1$, and $C D C 14 B$ ), DNA/RNA binding (e.g., HMGN4, TOPORS, and ESF1), actin cytoskeleton regulation (e.g., $D D N, M S N$, and CTNNA1), and extracellular matrix remodeling (e.g., COL4A2, IBSP) as well as cell differentiation into neuron (e.g., RAX2, OR11H12), bone (e.g., NIN, BMP15), endothelium/epithelium (e.g., MAGED2, CEACAM5), and hematopoietin (e.g., $H K 3, E P X)$. Importantly, the effect of EVs on the recovery from AKI was similar to the effect of MSCs, suggesting that the therapeutic effect by MSCs is mainly mediated by the MSC-derived EVs. In addition, pretreatment of RNase into MSC-derived EVs abolished the therapeutic effects by MSC-derived EVs, implying that RNAs from MSCs might be the main contributor for renal regeneration. Eirin et al. also characterized the mRNA cargo of EVs from porcine adipose tissue-derived MSCs by high-throughput RNA sequencing [27]. They revealed that EVs from MSCs contain mRNAs involved in transcription (e.g., MDFIC, POU3F1), angiogenesis (e.g., HES1, TCF4), adipogenesis (e.g., CEBPA, KLF7), and transforming growth factor- (TGF-) $\beta$ signaling (e.g., TGFB1, TGFB3). In comparison with these two studies of mRNA analysis (Bruno et al.: 43 genes; Eirin et al.: 182 genes), only 1 overlap of mRNA was observed [28], suggesting the possible heterogeneity of mRNAs of EVs from different types of MSCs.

\section{MicroRNAs in MSC-Derived Extracellular Vesicles for Renal Regeneration}

miRNAs are one of the non-protein-coding RNAs that regulate gene expressions. In mammals, miRNAs are predicted to control approximately $30 \%$ of all protein-coding genes and have shown to contribute to majority of cellular processes [29]. MSC-derived EVs contain a number of miRNAs. Ferguson et al. indicated the biological processes and pathways modulated by miRNAs from MSC-derived EVs using the NanoString profiling of miRNAs from EVs [30]. They revealed that enriched miRNAs regulate target gene transcriptions associated with Wnt signaling, profibrotic signaling via TGF- $\beta$ and PDGF, cell proliferation, and antiapoptosis. The top 23 miRNAs account for $79.1 \%$ of total miRNAs present in MSC-derived exosomes, and the remaining 148 miRNAs were at a very low ratio, suggesting that the top 23 miRNAs have predominant effects. These 23 miRNAs, miR-1246, miR-23a-3p, miR-451a, miR-125b-5p, miR-199a-3p/199b-3p, let-7a-5p, miR-4454/7975, miR-215p, let-7b-5p, miR-100-5p, miR-29a-3p, miR-144-3p, miR-29b-3p, miR-22-3p, miR-630, miR-221-3p, let-7i-5p, miR-424-5p, miR-191-5p, miR-25-3p, miR-130a-3p, miR$376 a-3 p$, and miR-27b-3p, were predicted to target 5481 genes using the microRNA Data Integration Portal (miRDIP). Among these miRNAs, miR-29, let-7, miR-451, miR-630, miR-191, miR-21, and miR-22 are overlapped in other reports on miRNA analysis from MSC-derived EVs [27, 31-38] (Table 2).
Although the role of miRNAs has just recently begun to be analyzed, emerging evidences indicate that MSC-derived miRNAs have essential roles on tissue regeneration. For example, the let-7 family has been shown to repress multiple genes involved in cell cycle, cell apoptosis, and cell proliferation, including CCNA2, CDC34, AURA/STK6, AURKB/ STK12, E2F5, and CDK8 [39]. In addition, the Let-7 family has been shown to switch macrophages to the M2-like profile by targeting the toll-like receptor (TLR) 4 [33] as well as the induction of osteogenic differentiation [36]. Furthermore, Wang et al. reported that the overexpression of let-7c from MSCs attenuates kidney injury and downregulates fibrotic markers, such as collagen IV $\alpha 1$, TGF- $\beta 1$, and TGF $\beta$ R1, in a unilateral ureteral obstruction (UUO) model [40]. miR125 has been reported to promote endothelial cell angiogenesis [41] while miR-29 has been reported to inhibit MCL-1 expression, an antiapoptotic protein [42], as well as ZFP36, which is an anti-inflammatory gene [43]. In fact, miR-29b inhibits the apoptotic pathway in doxorubicin-induced cardiotoxicity [44]. Furthermore, miR-29b attenuates angiotensin II-induced epithelial-mesenchymal transition (EMT) of rat renal tubular epithelial cells through the PI3K/AKT signaling pathway [45]. miR-21 is one of the miRNAs identified for the first time in mammals. miR-21 silences PTEN and GSK3b and reduces $\mathrm{NF} \kappa \mathrm{B}$ activity, which induces inflammation [46]. In addition, miR-21 ameliorates ischemia/reperfusion- (I/R-) induced AKI by preventing epithelial cell apoptosis and inhibiting the maturation of dendritic cells [47]. Taken together, miRNAs from MSCs might be one of the major contributors for promoting renal regeneration.

\section{Proteins in MSC-Derived Extracellular Vesicles for Renal Regeneration}

In addition to the soluble factors from MSCs, MSC-derived EVs contain proteins that directly transfer into recipient cells. Kim et al. analyzed the EV-contained proteins from human bone marrow MSCs and identified 730 proteins [48]. Using functional analysis by the Database for Annotation, Visualization and Integrated Discovery (DAVID) software, they indicated that these proteins are involved in cell proliferation, cell adhesion, cell migration, and the regulation of cell morphogenesis. They also highlighted the trophic proteins, including surface receptors (e.g., PDGFRB, EGFR, and PLAUR), signaling molecules (e.g., MAPK1, CDC42, RRAS/NRAS, and VAV2), and cell adhesion (e.g., EZR, FN1, IQGAP1, CD47, integrins, and LGALS1/ LGALS3) and MSC-associated proteins (e.g., CD9, CD63, CD81, CD109, CD151, CD248, and CD276). Another group also analyzed the proteins in EVs from human embryonic and bone marrow-derived MSCs, revealing that EVs contain trophic proteins associated with angiogenesis (VEGF, angiopoietin), inflammation (TNF-inducible gene 6 protein (TNFAIP6)), and TGF- $\beta$ signaling [49-51]. Taken together, MSC-derived EVs contain a number of trophic proteins that have the potential for the promotion of tissue regeneration. 
TABLE 2: miRNAs in MSC-derived EVs.

\begin{tabular}{|c|c|c|c|c|c|c|c|c|c|c|}
\hline $\begin{array}{l}\text { Reference } \\
\text { miRNA }\end{array}$ & [30] & [35] & {$[31]$} & {$[32]$} & [33] & [27] & {$[34]$} & {$[36]$} & [37] & [38] \\
\hline miR-1246 & 0 & & & & & & & & & \\
\hline miR-23a & O & & & & & & & & & \\
\hline miR-451a & 0 & & & 0 & & & & & 0 & \\
\hline miR-125b & O & & & & & & & & & \\
\hline miR-199a & 0 & & & & & & & & & \\
\hline let-7a & $\mathrm{O}$ & & & & & & & 0 & $\mathrm{O}$ & \\
\hline miR-4454/7975 & 0 & & & & & & & & & \\
\hline miR-21 & O & & & & & & O & & O & \\
\hline let-7b & 0 & & & & O & & & & & \\
\hline miR-100 & O & & & & & & & & & \\
\hline miR-29a & O & O & O & & & & & & & \\
\hline miR-144 & O & & & & & & & & & \\
\hline miR-29b & O & O & & & & & & & & \\
\hline miR-22 & O & & & & & & O & & & O \\
\hline miR-630 & O & & & O & & & & & & \\
\hline $\operatorname{miR}-221$ & O & & & & & & & & & \\
\hline let-7i & O & & & & & & & & & \\
\hline miR-424 & O & & & & & & & & & \\
\hline miR-191 & O & & & & & & O & & & \\
\hline miR-25 & O & & & & & & & & & \\
\hline miR-130a & O & & & & & & & & & \\
\hline miR-376a & O & & & & & & & & & \\
\hline $\mathrm{miR}-27 \mathrm{~b}$ & O & & & & & & & & & \\
\hline miR-30 & & O & & & & & & & & \\
\hline miR-210 & & O & & & & & & & & \\
\hline miR-24 & & & O & & & & & & & \\
\hline miR-1202 & & & & 0 & & & & & & \\
\hline miR-638 & & & & O & & & & & & \\
\hline miR-148a & & & & & & O & & O & & \\
\hline miR-532 & & & & & & O & & & & \\
\hline $\mathrm{miR}-378$ & & & & & & O & & & & \\
\hline let-7f & & & & & & O & & & & \\
\hline miR-486 & & & & & & & O & & & \\
\hline miR-10a & & & & & & & O & & & \\
\hline miR-10b & & & & & & & O & & & \\
\hline miR-222 & & & & & & & O & & & \\
\hline miR-143 & & & & & & & O & & O & \\
\hline miR-199b & & & & & & & & O & & \\
\hline miR-218 & & & & & & & & O & & \\
\hline miR-135b & & & & & & & & O & & \\
\hline miR-203 & & & & & & & & O & & \\
\hline miR-219 & & & & & & & & O & & \\
\hline miR-299 & & & & & & & & O & & \\
\hline miR-302b & & & & & & & & O & & \\
\hline miR-145 & & & & & & & & & O & \\
\hline miR-338 & & & & & & & & & O & \\
\hline miR-1260 & & & & & & & & & O & \\
\hline miR-1908 & & & & & & & & & $\mathrm{O}$ & \\
\hline
\end{tabular}




\section{MSC-Derived EV Therapy in Experimental AKI Models}

Emerging evidences have shown that treatment with MSCderived EVs attenuates renal injury after AKI in a variety of murine models (Table 3). For example, Bruno et al. revealed that human bone marrow MSC-derived EV injection improves renal function and tubular injury in a glycerol-induced AKI rat model through the stimulation of tubular cell proliferation and inhibition of cell apoptosis $[26,52]$. With the fact that the pretreatment with RNase into MSC-EVs reversed the therapeutic effects, these trophic mechanisms might be induced by RNAs in MSC-EVs. They also examined using another AKI model of cisplatin-induced AKI, indicating similar therapeutic effects including decreased renal cell apoptosis and preserved renal function [53].

Gatti et al. applied human bone marrow MSC-derived EVs into an I/R-induced AKI model, revealing that MSC-EV treatment attenuates I/R-induced AKI by reducing cell apoptosis and increasing renal tubular cell proliferation [54]. These trophic effects were abolished by RNase treatment similar to the study by Bruno et al. [26], reinforcing the concept that the trophic effect by MSC-EV treatment is mainly mediated by the mRNAs and/or miRNAs in MSC-EVs. Furthermore, they demonstrated that MSC-EV treatment also inhibits the progression of subsequent chronic kidney disease after AKI.

Zhou et al. showed that the injection of human umbilical cord MSC-derived EVs into the renal capsule attenuates cisplatin-induced AKI by improving oxidative stress as well as the inhibition of tubular cell apoptosis and necrosis [55]. Likewise, Zhang et al. reported that MSC-EV treatment protects against I/R-induced AKI through antioxidation possibly by enhancing NF-E2-related factor 2 (Nrf2)/antioxidant responsive element (ARE) activation [56]. They also reported that MSC-EV treatment ameliorates oxidative stress and renal injury in I/R-induced AKI through the decreased expression of NOX2 and reactive oxygen species (ROS) [57]. Furthermore, decreased renal fibrosis was observed with MSC-EV treatment. Zou et al. also reported decreased renal fibrosis with the treatment of human umbilical cord MSC-derived EVs as well as the downregulation of CXCL1 and decrease of CD68 ${ }^{+}$ macrophage [58]. They also reported the increased expression of renal VEGF with the treatment of MSC-EVs as well as decreased renal fibrosis, indicating that MSC-derived EVs affect angiogenesis [59]. Importantly, the pretreatment with RNase into MSC-EVs abolished the trophic effects [59].

Reis et al. reported that rat bone marrow MSC-derived $\mathrm{EV}$ treatment improves renal function in gentamicininduced AKI by increasing tubular cell proliferation, suppressing cell apoptosis and necrosis, and inhibiting renal inflammation [60]. With MSC-EV treatment, proinflammatory cytokines decrease while anti-inflammatory cytokines increase. Likewise, Wang et al. reported that MSC-EV treatment ameliorates I/R-induced AKI by reducing inflammatory cytokines such as IL- $\beta$ and TNF- $\alpha$ [61], indicating that the regulation of inflammation is one of the major mechanisms for renal protection by MSC-EVs.

$\mathrm{Ju}$ et al. reported that human umbilical cord MSCderived EV treatment protects from I/R-induced AKI by increasing ERK1/2 expression and HGF, which promotes tubular cell dedifferentiation and growth [62]. RNase treatment abolishes these trophic effects, suggesting that RNAs in MSC-EV are essential factors for renoprotection. Gu et al. reported that human umbilical cord MSC-derived EV treatment attenuated AKI-induced renal injury by preserving mitochondrial morphology that was paralleled with reduced apoptosis. They also revealed that miR-30 antagomirs dramatically reduced these protective effects [63]. Furthermore, Collino et al. reported that miRNA deletion in MSCs and EVs reduces regenerative effects in glycerol-induced AKI [64]. These reports imply the critical role of miRNAs in MSC-EVs on promoting renal protection and regeneration after AKI.

Wang et al. reported improved renal function with the treatment of umbilical cord MSC-derived EVs in cisplatininduced AKI [65]. They revealed that MSC-EV treatment prevented renal injury through the activation of autophagy, and the effect was abolished with the autophagy inhibitor, 3methyladenine. In the same line, Jia et al. recently reported that umbilical cord MSC-derived EV treatment prevents cisplatin-induced renal injury through the activation of autophagy via trophic factor $14-3-3 \zeta$ which interacts with ATG-16L [66], indicating proteins in MSC-EVs also contribute to renal protection. Taken together, the activation of autophagy is one of the important mechanisms by MSC-EV treatment.

In addition to the bone marrow-derived and umbilical cord-derived MSC analyses, MSCs from different origins were also examined. Choi et al. applied EVs from kidneyderived MSCs in I/R-induced AKI mice, revealing the trophic effects through the increased cell proliferation as well as the inhibition of cell apoptosis [67]. Lin et al. applied adiposederived MSCs in I/R-induced AKI and revealed that the combination of adipose-derived MSC-EVs and MSC treatment protects from renal injury after AKI through the inhibition of cell apoptosis, oxidative stress, and renal fibrosis [68]. These reports suggest MSC-EVs from different origins also provide renal protection and regeneration.

Taken together, renoprotective effects by MSC-derived secretomes might be mainly mediated by EV-containing mRNA, miRNA, and proteins through a variety of mechanisms. Importantly, these trophic mechanisms from MSCs can be divided into two types, renal protection and regeneration after injury. Renal protection is mainly mediated through the suppression of cell apoptosis, cell necrosis, renal fibrosis, renal inflammation, and oxidative stress as well as the promotion of autophagy. Regeneration is mediated through the increase in cell proliferation, migration, tubular cell dedifferentiation, and angiogenesis. Secretomes from MSCs and the trophic mechanisms against renal injury are summarized (Figure 1). Further analyses are required to elucidate the detailed mechanisms by which MSC-EVs protect the kidney from AKI.

\section{Heterogeneity of Secretomes from MSCs by Different Origins}

While a number of studies have identified the secretomes from MSCs for renal protection, the origin of MSCs might affect the types and quantities of trophic secretomes. As 


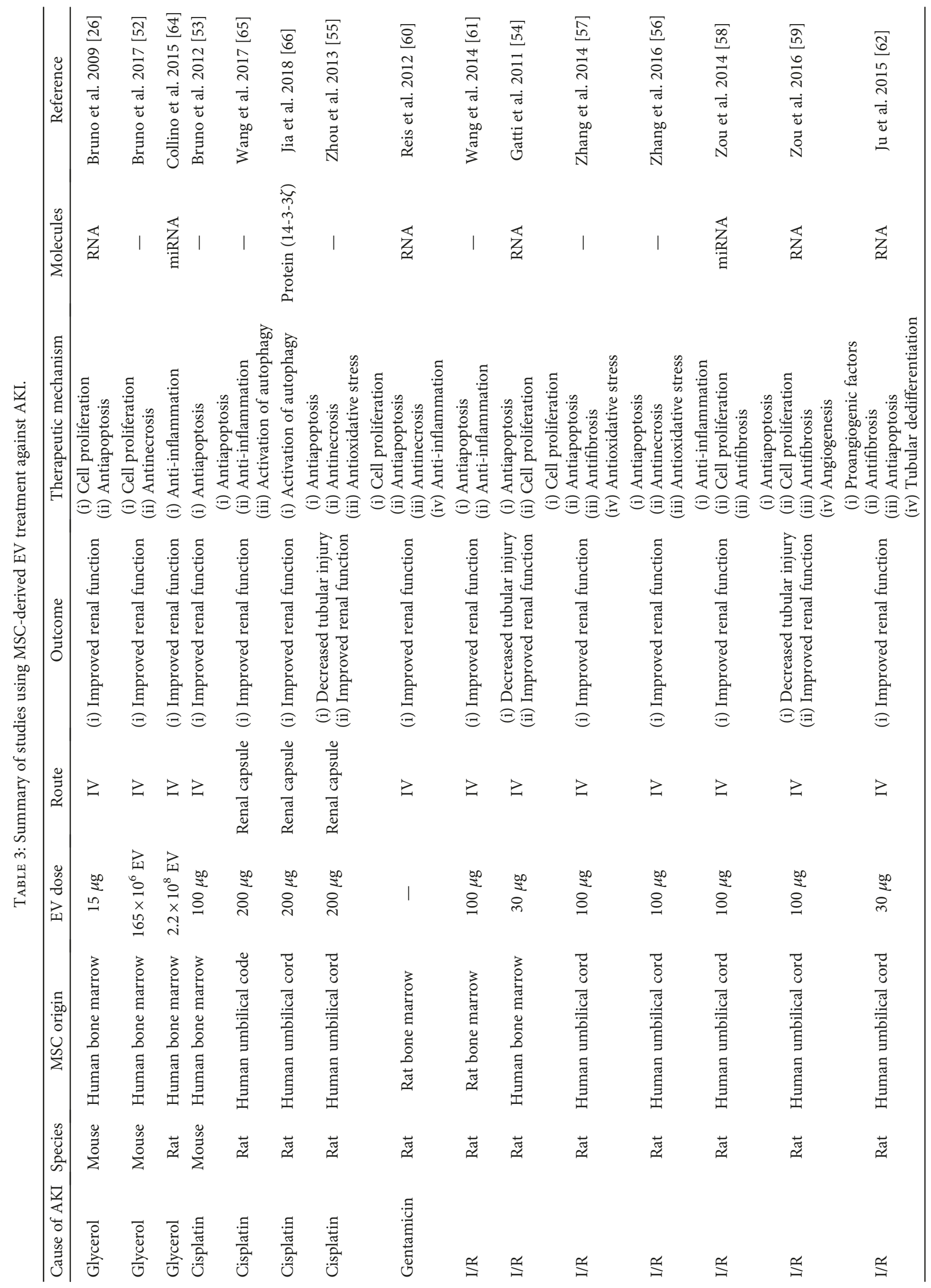




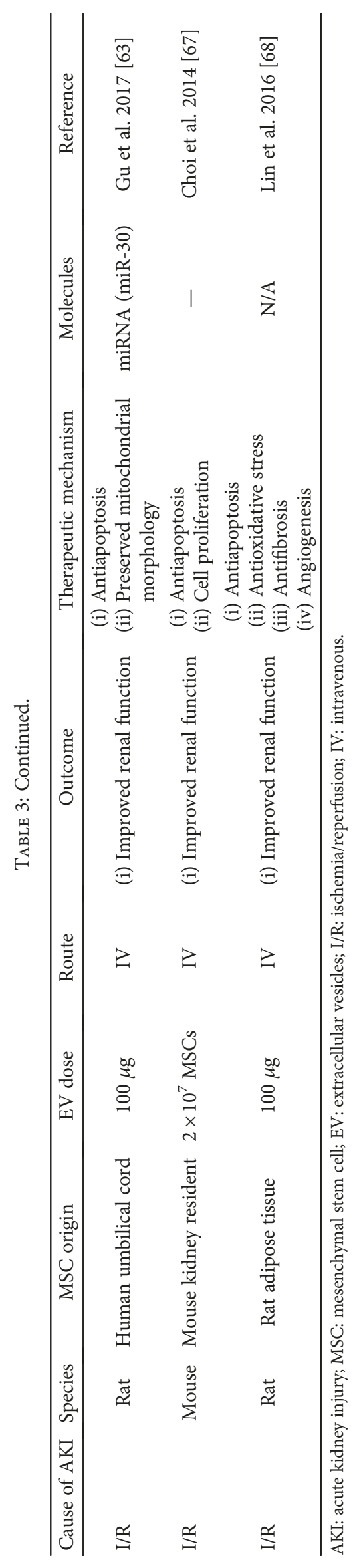



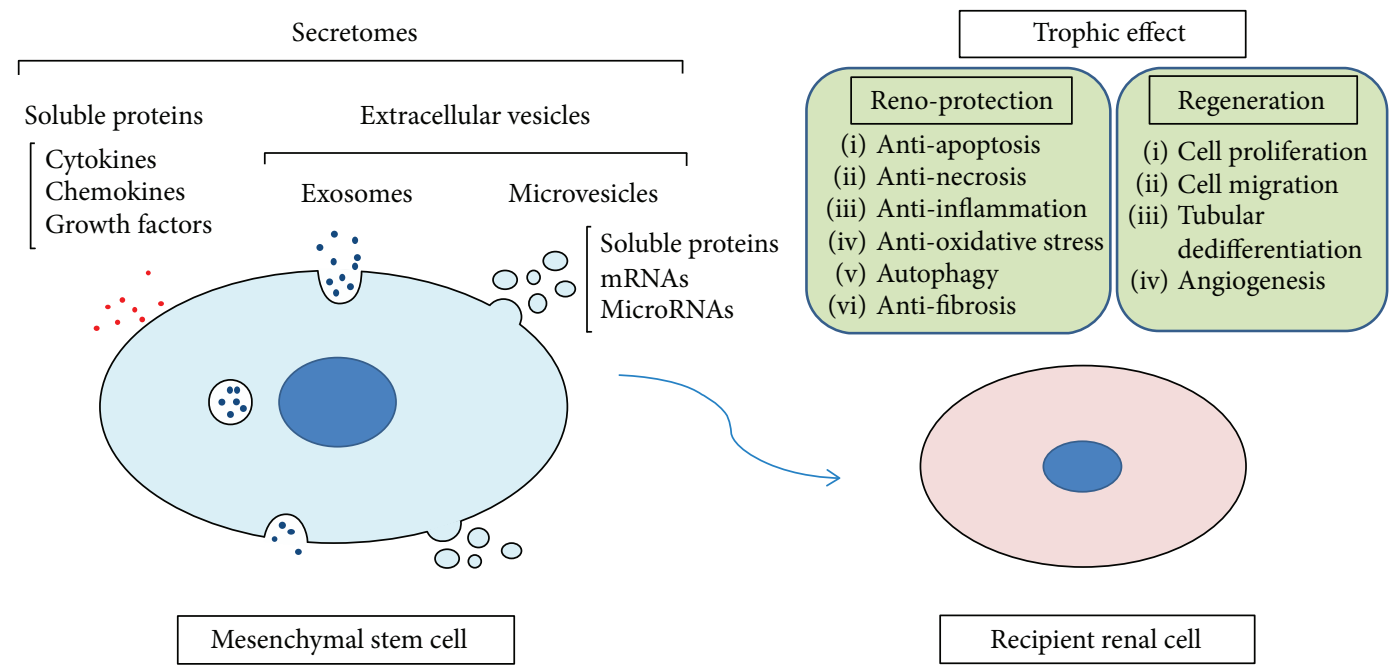

FIGURE 1: Schema of trophic mechanisms via secretomes from mesenchymal stem cells.

described above, Nargesi et al. reported the heterogeneity of mRNAs in MSC-EVs from bone marrow-derived MSCs and adipose-derived MSCs. They indicate that less than $3 \%$ of mRNA expression is overlapped between these 2 types of MSCs [28]. Lindsay et al. reported that human MSCs from the olfactory mucosa but not from the bone marrow enhance central nervous system myelination, implying the difference of secretomes from MSCs from different origins [69]. They also reported the different quantities of miRNAs between human olfactory mucosa-derived MSCs and bone marrow-derived MSCs [70]. Using the analysis of miRNA analysis, they showed that $64 \%$ of miRNAs isolated from both MSCs were equivalently expressed while 26 miRNAs showed different amounts of expressions, especially in the expression of miR-140-5p and miR-146a-5p, which regulate inflammatory cytokines, such as CXCL12, IL-6, IL-8, and CCL2. These data strongly suggest the heterogeneity in the point of secretomes from different sources of MSCs, which may affect the regenerative effect in MSC-based therapy. Because miRNAs are known to act as regulatory signals for maintaining stemness, self-renewal, and differentiation in adult stem cells, the characterization of miRNAs from MSCs from different tissues may give us insight into what makes the different biological activities [71]. Indeed, it is known that the signaling pathways involved in cell fate specification, including Wnt, BMP, Notch, and TGF- $\beta$ pathways, are associated with MSC differentiations [72-74]. miRNAs are reported to control the balance between self-renewal and differentiation [75]. Lazzarini et al. reported that MSCs isolated from skin and amniotic fluid shared the expression of core miRNAs related to stemness and authorize the definition of MSCs while there were significant differences in the expression of miRNAs associated with adipogenesis, indicating the existence of tissue specificity [76].

Although MSCs from different origins have similarities, characteristic differences have been reported. For example, placental MSCs have superior migratory capacity but less adipogenic potential $[77,78]$. Umbilical cord-derived MSCs do not express tumor-associated fibroblast phenotypes [79] and thus have no opportunity to grow tumors. Adiposederived MSCs possess a higher potential for angiogenesis and vasculogenesis as well as adipogenesis [80]. Tsai et al. applied a microarray comparison between amniotic fluid-, amniotic membrane-, and cord blood-derived MSCs, revealing specific biological functions for MSCs from different origins [81]. In addition, Pelekanos et al. compared MSC-like cells from the heart, bone marrow, and kidney [82] and reported that these 3 types of MSCs share morphological and molecular characteristics as well as multipotency while there are differences in the expression of organ-specific genes to maintain the "memory of tissue origin" reflective of their unique ontogeny and functional roles. They revealed the increased expressions of Mylk, Myom, Desmin, and Serpinb2 in kidney-derived MSCs, suggesting a strong relationship with the perivascular and mesangial cells, indicating kidney-specific gene expressions. Taken together, there might be distinct functional roles of MSCs isolated from different tissues.

What makes the differences in MSCs from different origins? One of the essential differences between MSCs from different origins might be their cellular niche. The fate of stem cells might be regulated by their microenvironment [83], which might provide the tissue specificity. The transmembrane cell adhesion proteins, cadherins, act in cell-cell adhesion, differentiation, and polarity in MSCs and interact with Wnt, which are involved in the MSC niche [83]. The location of MSCs is associated with how they interact with these molecules, and it would affect their functions. Because MSCs interact with other niche cells both locally and systemically [84], the difference of MSC niche might make the difference of secretomes from MSCs. In addition, the bone marrow milieu is hypoxic in nature. The oxygenic difference also makes the characteristic change of MSCs from the bone marrow and others.

Despite the heterogeneity of secretomes from MSCs isolated from different tissues, there are few papers focusing on the different secretomes of MSCs from different origins. Further analysis and comparison are necessary for elucidating the heterogeneity of secretomes from different MSCs. 
TABLE 4: Summary of studies about secretome changes from MSCs in different stimuli.

\begin{tabular}{|c|c|c|}
\hline Stimuli & Increased secretomes & Reference \\
\hline Hypoxia & $\begin{array}{l}\text { Tropomyosin, VEGF, SDF-1, FGF-2, HGF, } \\
\text { IGF-1, Oct-4, Rex-1, and IDO }\end{array}$ & {$[88,90-93]$} \\
\hline IFN- $\gamma$ & IDO, PGE-2, and Gal-9 & {$[93-96]$} \\
\hline TNF- $\alpha$ & Gal-9, BMP-2, VEGF, SDF-1, FGF-2, HGF, and IGF-1 & {$[91,96,97]$} \\
\hline TLR signal & Gal-9 & {$[96]$} \\
\hline $\begin{array}{l}\text { Inflammatory stimuli } \\
\text { (IL-1 } \beta \text {, IL-6, IL-20, and TNF- } \alpha \text { ) }\end{array}$ & $\begin{array}{l}\text { CXCL-16, GRO, ENA-78, MIP-1-delta, osteoprotegerin, } \\
\text { MCP-1, MCP-2, MCP-3, } \\
\text { IL-6, GCP-2, and IL-2RA }\end{array}$ & [98] \\
\hline LPS & VEGF, SDF-1, FGF-2, HGF, and IGF-1 & {$[91]$} \\
\hline $3 \mathrm{D}$ culture & TSG-6, STC1, TRIL, IL-24, and CD82 & {$[100-102]$} \\
\hline Nanosilicate & More than 4000 gene expression change & [103] \\
\hline Aging & $\begin{array}{c}\text { Increase: miR-335 } \\
\text { Decrease: TNFR, IFNGR, CCR7, miR-146a, miR-155, and miR-132 }\end{array}$ & {$[85,86]$} \\
\hline \multirow{4}{*}{ TGF- $\beta$} & Cytoskeletal factors (e.g. T-platin, gelsolin) & \multirow{4}{*}[99]{} \\
\hline & Matrix synthesis factors (e.g., collagen-binding protein 2) & \\
\hline & Membrane proteins (e.g., annexin A6, annexin A2) & \\
\hline & $\begin{array}{c}\text { Metabolic enzymes (e.g., thioredoxin reductase, transaldolase, } \\
\text { and malate dehydrogenase) }\end{array}$ & \\
\hline
\end{tabular}

LPS: lipopolysaccharide; 3D: 3-dimensional.

\section{Inducible Secretomes from MSCs by Different Stimuli}

In addition to the difference of MSC origin, increasing evidences have proposed the changes of secretomes by different stimuli into MSCs that include aging, hypoxia, inflammatory stimuli, 3-dimensional (3D) culture condition, microparticle stimuli, nanosilicate stimuli, and TGF- $\beta$ stimuli (Table 4). Bustos et al. demonstrated the age-dependent decrease in the expressions of several cytokine/chemokine receptors, which diminish the cell migration and activation of MSCs [85]. They showed the decreased expressions of TNFR, IFNGR, and CCR7 in aged bone marrow-derived MSCs, which might result in the decrease in protective potential. Fafián-Labora et al. explored the difference in the point of miRNA expressions in MSC-EVs, revealing the age-dependent decrease in miR-146a, miR-155, and miR132 expression and increase in miR-335 expression [86].

Hypoxic condition affects a number of gene transcriptions via stabilization of HIF- $1 \alpha$, including angiogenic factors such as VEGF [87]. Song et al. performed the proteomic analysis of MSC secretomes under hypoxia [88], revealing the significant difference in the expressions of 66 out of 231 proteins between the normoxic and hypoxic conditioned media. Especially, they indicated the dramatic increase in 2 tropomyosin isoform expressions in hypoxic condition in a HIF- $1 \alpha$ dependent manner. They hypothesize according to the GeneMANIA network analysis that tropomyosin might activate NOS3, which is known to protect against ischemic injury [89]. In addition, Ceradini et al. reported that HIF$1 \alpha$-induced SDF-1 expression in MSCs mediates the recruitments of MSCs to the sites of injured tissue [90]. Furthermore, Crisostomo et al. reported the increased expressions of FGF-2, HGF, and IGF-1 from bone marrow-derived MSCs as well as VEGF in an NF $\kappa \mathrm{B}$-dependent manner under hypoxia [91]. In addition to the secretomes from MSCs, hypoxic condition keeps MSCs in an undifferentiated phenotype for self-renewal. Hypoxic condition increases the expression of stem cell markers, such as Oct- 4 and Rex-1 from MSCs, indicating the increased stemness [92]. Hypoxic condition also affects the secretomes related to inflammation. Munn and Mellor reported that the hypoxic condition increases the expression of IDO from MSCs, which regulates the immune system through limiting $\mathrm{T}$ cell function and engaging mechanisms of immune tolerance [93]. In summary, hypoxic condition increases the secretomes from MSCs that are associated with angiogenesis and inflammation.

Inflammatory stimuli also affect the secretomes from MSCs. For example, IFN- $\gamma$ treatment into MSCs increases IDO expression [94], which results in the inhibition of MSC cell proliferation as well as the inhibition of the differentiation potential of MSCs into neuron and adipocyte. IFN- $\gamma$ also upregulates the expression of PGE-2 [95]. Inflammatory stimuli by IFN- $\gamma$, TNF- $\alpha$, and TLR signals increase the expressions of Gal-9 from MSCs [96]. TNF- $\alpha$ also increases the expression of BMP-2, which leads to the increase in cell proliferation, migration, and osteogenic differentiation [97]. Like hypoxic condition, TNF- $\alpha$ and lipopolysaccharide (LPS) treatments increase the expressions of VEGF, FGF-2, HGF, and IGF-1 from MSCs [91]. Xing et al. reported that inflammatory stimuli increase chemokine secretion to promote the MSC recruiting capacity [98]. They showed the significant increase in cytokine and chemokine expressions including CXCL-16, GRO, ENA-78, MIP-1-delta, osteoprotegerin, MCP-1, MCP-2, MCP-3, IL-6, GCP-2, and IL-2R $\alpha$ in proinflammatory treated (IL- $1 \beta$, IL-6, IL-20, and TNF- $\alpha$ ) culture supernatant compared to nontreated culture supernatant during MSC culture. They 
also indicated the increased migration of MSCs in the proinflammatory factor-treated culture medium, suggesting that increased soluble factors affect the recruiting ability of MSCs. Overall, inflammatory stimuli increase the regenerative process through the enhanced release of secretomes from MSCs.

Wang et al. applied proteomic profiling to explore the expression pattern of soluble proteins from MSCs with the stimuli of TGF- $\beta 1$ [99], revealing the secreting differences in around 30 proteins with TGF- $\beta 1$ treatment, including cytoskeletal factors (e.g., T-platin, gelsolin), matrix synthesis factors (e.g., collagen-binding protein 2), membrane proteins (e.g., annexin A6, annexin A2), and metabolic enzymes (e.g., thioredoxin reductase, transaldolase, and malate dehydrogenase). They also demonstrated that the decreased expression of gelsolin with TGF- $\beta 1$ treatment enhances the assembly of $\alpha$-actin and actin filaments, which might lead to MSC differentiation. Hence, we need to point out that these different stimuli not only affect the secretomes from MSCs but also affect MSC differentiation.

3D culture systems, such as spheroid culture also, affect the secretomes from MSCs. Bartosh et al. reported the increased expressions of TSG-6 as well as STC1 and three anticancer proteins, TRIL, IL-24 and CD82, from MSCs when they are grown in 3D spheroids [100]. They also revealed that the assembly of MSC into spheres triggers caspase-dependent IL-1 signaling and the secretion of modulators of inflammation [101]. In addition, Frith et al. indicated the increased expression of IL-24 from MSCs cultured in dynamic 3D culture condition compared to monolayer culture condition [102]. These data indicate the importance of the niche and/or environment of culture condition in the point of secretomes from MSCs.

More recently, Carrow et al. reported that human MSCs show widespread change of secretomes when they are stimulated by two-dimensional nanosilicates [103]. They revealed the change of more than 4000 gene expressions by nanosilicate exposure using high-throughput sequencing (RNAseq). Nanosilicate attaches to the cell membrane, internalizes the cells, and activates stress-response pathways including MAPK, which also affects MSC differentiation toward bone and chondrogenic tissue. This information about the change of secretomes with different stimuli is important for the stable and high-quality supply of secretomes for the cell-free therapy by MSC-EVs against tissue injuries.

\section{Conclusion}

In this review article, we summarize the current evidence about the secretomes from MSCs and the therapeutic mechanisms against AKI. There are several advantages with the use of MSC-EVs. MSC-EVs are the cell-free sources for tissue regeneration which might be safer rather than using MSC cells themselves. In addition, EVs are the sources for cellcell communications, which might be easily transferred into recipient cells with lower concentration of the factors. Because the renoprotective effect of MSCs against AKI is mainly mediated by MSC-EVs, it may open a new strategy to treat against AKI. There might be the heterogeneity of secretomes of MSCs that depend on the cell origin of MSCs and different stimuli, including hypoxia, inflammatory signals, and aging. Therefore, we need to explore the change of secretomes and further specify the trophic factors, so that we can identify the best sources and factors for the therapy with MSC-EVs. It can also help to get stable and high-quality secretomes from MSCs. In summary, MSC$\mathrm{EV}$ treatment is the promising therapeutic option for the renal protection and regeneration after AKI. Although further analysis and experiments are necessary to develop this therapy, it would open a new strategy to treat against AKI.

\section{Conflicts of Interest}

The authors declare that there is no conflict of interest regarding the publication of this paper.

\section{Acknowledgments}

This work was supported by the Japan Society for the Promotion of Science (JSPS)/Grant-in-Aid for Young Scientists (18K15978).

\section{References}

[1] S. A. Silver, Z. Harel, E. McArthur et al., "Causes of death after a hospitalization with AKI," Journal of the American Society of Nephrology, vol. 29, no. 3, pp. 1001-1010, 2018.

[2] R. K. Hsu and C. Y. Hsu, "The role of acute kidney injury in chronic kidney disease," Seminars in Nephrology, vol. 36, no. 4, pp. 283-292, 2016.

[3] L. G. Forni, M. Darmon, M. Ostermann et al., "Renal recovery after acute kidney injury," Intensive Care Medicine, vol. 43, no. 6, pp. 855-866, 2017.

[4] M. F. Pittenger, A. M. Mackay, S. C. Beck et al., "Multilineage potential of adult human mesenchymal stem cells," Science, vol. 284, no. 5411, pp. 143-147, 1999.

[5] M. Broekema, M. C. Harmsen, J. A. Koerts et al., "Determinants of tubular bone marrow-derived cell engraftment after renal ischemia/reperfusion in rats," Kidney International, vol. 68, no. 6, pp. 2572-2581, 2005.

[6] A. I. Hoch, B. Y. Binder, D. C. Genetos, and J. K. Leach, "Differentiation-dependent secretion of proangiogenic factors by mesenchymal stem cells," PLoS One, vol. 7, no. 4, article e35579, 2012.

[7] A. Oskowitz, H. McFerrin, M. Gutschow, M. L. Carter, and R. Pochampally, "Serum-deprived human multipotent mesenchymal stromal cells (MSCs) are highly angiogenic," Stem Cell Research, vol. 6, no. 3, pp. 215-225, 2011.

[8] S. K. Sze, D. P. V. de Kleijn, R. C. Lai et al., "Elucidating the secretion proteome of human embryonic stem cell-derived mesenchymal stem cells," Molecular \& Cellular Proteomics, vol. 6, no. 10, pp. 1680-1689, 2007.

[9] H. Zhang, R. Yang, Z. Wang, G. Lin, T. F. Lue, and C. S. Lin, "Adipose tissue-derived stem cells secrete CXCL5 cytokine with neurotrophic effects on cavernous nerve regeneration," The Journal of Sexual Medicine, vol. 8, no. 2, pp. 437-446, 2011.

[10] C. Liu and S. Hwang, "Cytokine interactions in mesenchymal stem cells from cord blood," Cytokine, vol. 32, no. 6, pp. 270279, 2005. 
[11] C. A. Ribeiro, J. S. Fraga, M. Graos et al., "The secretome of stem cells isolated from the adipose tissue and Wharton jelly acts differently on central nervous system derived cell populations," Stem Cell Research \& Therapy, vol. 3, no. 3, p. 18, 2012.

[12] G. E. Kilroy, S. J. Foster, X. Wu et al., "Cytokine profile of human adipose-derived stem cells: expression of angiogenic, hematopoietic, and pro-inflammatory factors," Journal of Cellular Physiology, vol. 212, no. 3, pp. 702-709, 2007.

[13] J. A. Potian, H. Aviv, N. M. Ponzio, J. S. Harrison, and P. Rameshwar, "Veto-like activity of mesenchymal stem cells: functional discrimination between cellular responses to alloantigens and recall antigens," Journal of Immunology, vol. 171, no. 7, pp. 3426-3434, 2003.

[14] A. I. Caplan and J. E. Dennis, "Mesenchymal stem cells as trophic mediators," Journal of Cellular Biochemistry, vol. 98, no. 5, pp. 1076-1084, 2006.

[15] M. Groh, B. Maitra, E. Szekely, and O. Koc, "Human mesenchymal stem cells require monocyte-mediated activation to suppress alloreactive T cells," Experimental Hematology, vol. 33, no. 8, pp. 928-934, 2005.

[16] F. Togel, Z. Hu, K. Weiss, J. Isaac, C. Lange, and C. Westenfelder, "Administered mesenchymal stem cells protect against ischemic acute renal failure through differentiation-independent mechanisms," American Journal of Physiology. Renal Physiology, vol. 289, no. 1, pp. F31-F42, 2005.

[17] S. Aggarwal and M. F. Pittenger, "Human mesenchymal stem cells modulate allogeneic immune cell responses," Blood, vol. 105, no. 4, pp. 1815-1822, 2005.

[18] C. Rota, B. Imberti, M. Pozzobon et al., "Human amniotic fluid stem cell preconditioning improves their regenerative potential," Stem Cells and Development, vol. 21, no. 11, pp. 1911-1923, 2012.

[19] S. Lv, G. Liu, A. Sun et al., "Mesenchymal stem cells ameliorate diabetic glomerular fibrosis in vivo and in vitro by inhibiting TGF- $\beta$ signalling via secretion of bone morphogenetic protein 7," Diabetes \& Vascular Disease Research, vol. 11, no. 4, pp. 251-261, 2014.

[20] M. Riazifar, E. J. Pone, J. Lotvall, and W. Zhao, "Stem cell extracellular vesicles: extended messages of regeneration," Annual Review of Pharmacology and Toxicology, vol. 57, no. 1, pp. 125-154, 2017.

[21] M. Yáñez-Mó, P. R.-M. Siljander, Z. Andreu et al., "Biological properties of extracellular vesicles and their physiological functions," Journal of Extracellular Vesicles, vol. 4, no. 1, p. $27066,2015$.

[22] M. Colombo, G. Raposo, and C. Thery, "Biogenesis, secretion, and intercellular interactions of exosomes and other extracellular vesicles," Annual Review of Cell and Developmental Biology, vol. 30, no. 1, pp. 255-289, 2014.

[23] F. Rad, A. A. Pourfathollah, F. Yari, S. Mohammadi, and M. Kheirandish, "Microvesicles preparation from mesenchymal stem cells," Medical Journal of the Islamic Republic of Iran, vol. 30, p. 398, 2016.

[24] A. O. Batagov and I. V. Kurochkin, "Exosomes secreted by human cells transport largely mRNA fragments that are enriched in the $3^{\prime}$-untranslated regions," Biology Direct, vol. 8, no. 1, p. 12, 2013.

[25] H. Valadi, K. Ekstrom, A. Bossios, M. Sjostrand, J. J. Lee, and J. O. Lotvall, "Exosome-mediated transfer of mRNAs and
microRNAs is a novel mechanism of genetic exchange between cells," Nature Cell Biology, vol. 9, no. 6, pp. 654659, 2007.

[26] S. Bruno, C. Grange, M. C. Deregibus et al., "Mesenchymal stem cell-derived microvesicles protect against acute tubular injury," Journal of the American Society of Nephrology, vol. 20, no. 5, pp. 1053-1067, 2009.

[27] A. Eirin, S. M. Riester, X. Y. Zhu et al., "MicroRNA and mRNA cargo of extracellular vesicles from porcine adipose tissue-derived mesenchymal stem cells," Gene, vol. 551, no. 1, pp. 55-64, 2014.

[28] A. A. Nargesi, L. O. Lerman, and A. Eirin, "Mesenchymal stem cell-derived extracellular vesicles for renal repair," Current Gene Therapy, vol. 17, no. 1, pp. 29-42, 2017.

[29] W. Filipowicz, S. N. Bhattacharyya, and N. Sonenberg, "Mechanisms of post-transcriptional regulation by microRNAs: are the answers in sight?," Nature Reviews. Genetics, vol. 9, no. 2, pp. 102-114, 2008.

[30] S. W. Ferguson, J. Wang, C. J. Lee et al., “The microRNA regulatory landscape of MSC-derived exosomes: a systems view," Scientific Reports, vol. 8, no. 1, p. 1419, 2018.

[31] L. Shao, Y. Zhang, B. Lan et al., "MiRNA-sequence indicates that mesenchymal stem cells and exosomes have similar mechanism to enhance cardiac repair," BioMed Research International, vol. 2017, Article ID 4150705, 9 pages, 2017.

[32] D. G. Phinney, M. Di Giuseppe, J. Njah et al., "Mesenchymal stem cells use extracellular vesicles to outsource mitophagy and shuttle microRNAs," Nature Communications, vol. 6, no. 1, p. 8472, 2015.

[33] D. Ti, H. Hao, C. Tong et al., "LPS-preconditioned mesenchymal stromal cells modify macrophage polarization for resolution of chronic inflammation via exosome-shuttled let-7b," Journal of Translational Medicine, vol. 13, no. 1, p. 308, 2015.

[34] S. R. Baglio, K. Rooijers, D. Koppers-Lalic et al., "Human bone marrow- and adipose-mesenchymal stem cells secrete exosomes enriched in distinctive miRNA and tRNA species," Stem Cell Research \& Therapy, vol. 6, no. 1, p. 127, 2015.

[35] J. He, Y. Wang, X. Lu et al., "Micro-vesicles derived from bone marrow stem cells protect the kidney both in vivo and in vitro by microRNA-dependent repairing," Nephrology, vol. 20, no. 9, pp. 591-600, 2015.

[36] J. F. Xu, G. H. Yang, X. H. Pan et al., “Altered microRNA expression profile in exosomes during osteogenic differentiation of human bone marrow-derived mesenchymal stem cells," PLoS One, vol. 9, no. 12, article e114627, 2014.

[37] M. Garcia-Contreras, C. D. Vera-Donoso, J. M. HernandezAndreu, J. M. Garcia-Verdugo, and E. Oltra, "Therapeutic potential of human adipose-derived stem cells (ADSCs) from cancer patients: a pilot study," PLoS One, vol. 9, no. 11, article e113288, 2014.

[38] Y. Feng, W. Huang, M. Wani, X. Yu, and M. Ashraf, "Ischemic preconditioning potentiates the protective effect of stem cells through secretion of exosomes by targeting Mecp2 via miR-22," PLoS One, vol. 9, no. 2, article e88685, 2014.

[39] C. D. Johnson, A. Esquela-Kerscher, G. Stefani et al., "The let-7 microRNA represses cell proliferation pathways in human cells," Cancer Research, vol. 67, no. 16, pp. 77137722, 2007. 
[40] B. Wang, K. Yao, B. M. Huuskes et al., "Mesenchymal stem cells deliver exogenous MicroRNA-let7c via exosomes to attenuate renal fibrosis," Molecular Therapy, vol. 24, no. 7, pp. 1290-1301, 2016.

[41] X. Liang, L. Zhang, S. Wang, Q. Han, and R. C. Zhao, "Exosomes secreted by mesenchymal stem cells promote endothelial cell angiogenesis by transferring miR-125a," Journal of Cell Science, vol. 129, no. 11, pp. 2182-2189, 2016.

[42] J. L. Mott, S. Kobayashi, S. F. Bronk, and G. J. Gores, "mir-29 regulates Mcl-1 protein expression and apoptosis," Oncogene, vol. 26, no. 42, pp. 6133-6140, 2007.

[43] C. A. Gebeshuber, K. Zatloukal, and J. Martinez, "miR-29a suppresses tristetraprolin, which is a regulator of epithelial polarity and metastasis," EMBO Reports, vol. 10, no. 4, pp. 400-405, 2009.

[44] X. Jing, J. Yang, L. Jiang, J. Chen, and H. Wang, "MicroRNA$29 \mathrm{~b}$ regulates the mitochondria-dependent apoptotic pathway by targeting Bax in doxorubicin cardiotoxicity," Cellular Physiology and Biochemistry, vol. 48, no. 2, pp. 692-704, 2018.

[45] H. Hu, S. Hu, S. Xu, Y. Gao, F. Zeng, and H. Shui, "miR-29b regulates Ang II-induced EMT of rat renal tubular epithelial cells via targeting PI3K/AKT signaling pathway," International Journal of Molecular Medicine, vol. 42, no. 1, pp. 453-460, 2018.

[46] A. Das, K. Ganesh, S. Khanna, C. K. Sen, and S. Roy, "Engulfment of apoptotic cells by macrophages: a role of microRNA21 in the resolution of wound inflammation," Journal of Immunology, vol. 192, no. 3, pp. 1120-1129, 2014.

[47] N. Song, T. Zhang, X. Xu et al., "miR-21 protects against ischemia/reperfusion-induced acute kidney injury by preventing epithelial cell apoptosis and inhibiting dendritic cell maturation," Frontiers in Physiology, vol. 9, 2018.

[48] H. S. Kim, D. Y. Choi, S. J. Yun et al., "Proteomic analysis of microvesicles derived from human mesenchymal stem cells," Journal of Proteome Research, vol. 11, no. 2, pp. 839-849, 2012.

[49] A. Eirin, X. Y. Zhu, A. S. Puranik et al., "Comparative proteomic analysis of extracellular vesicles isolated from porcine adipose tissue-derived mesenchymal stem/stromal cells," Scientific Reports, vol. 6, no. 1, article 36120, 2016.

[50] J. D. Anderson, H. J. Johansson, C. S. Graham et al., "Comprehensive proteomic analysis of mesenchymal stem cell exosomes reveals modulation of angiogenesis via nuclear factor-KappaB signaling," Stem Cells, vol. 34, no. 3, pp. 601-613, 2016.

[51] R. C. Lai, S. S. Tan, B. J. Teh et al., "Proteolytic potential of the MSC exosome proteome: implications for an exosomemediated delivery of therapeutic proteasome," International Journal of Proteomics, vol. 2012, Article ID 971907, 14 pages, 2012.

[52] S. Bruno, M. Tapparo, F. Collino et al., "Renal regenerative potential of different extracellular vesicle populations derived from bone marrow mesenchymal stromal cells," Tissue Engineering. Part A, vol. 23, no. 21-22, pp. 12621273, 2017.

[53] S. Bruno, C. Grange, F. Collino et al., "Microvesicles derived from mesenchymal stem cells enhance survival in a lethal model of acute kidney injury," PLoS One, vol. 7, no. 3, article e33115, 2012.
[54] S. Gatti, S. Bruno, M. C. Deregibus et al., "Microvesicles derived from human adult mesenchymal stem cells protect against ischaemia-reperfusion-induced acute and chronic kidney injury," Nephrology, Dialysis, Transplantation, vol. 26, no. 5, pp. 1474-1483, 2011.

[55] Y. Zhou, H. Xu, W. Xu et al., "Exosomes released by human umbilical cord mesenchymal stem cells protect against cisplatin-induced renal oxidative stress and apoptosis in vivo and in vitro," Stem Cell Research \& Therapy, vol. 4, no. 2, p. 34, 2013.

[56] G. Zhang, X. Zou, Y. Huang et al., "Mesenchymal stromal cell-derived extracellular vesicles protect against acute kidney injury through anti-oxidation by enhancing Nrf2/ARE activation in rats," Kidney \& Blood Pressure Research, vol. 41, no. 2, pp. 119-128, 2016.

[57] G. Zhang, X. Zou, S. Miao et al., "The anti-oxidative role of micro-vesicles derived from human Wharton-Jelly mesenchymal stromal cells through NOX2/gp91(phox) suppression in alleviating renal ischemia-reperfusion injury in rats," PLoS One, vol. 9, no. 3, article e92129, 2014.

[58] X. Zou, G. Zhang, Z. Cheng et al., "Microvesicles derived from human Wharton's Jelly mesenchymal stromal cells ameliorate renal ischemia-reperfusion injury in rats by suppressing CX3CL1," Stem Cell Research \& Therapy, vol. 5, no. 2, p. 40, 2014.

[59] X. Zou, D. Gu, X. Xing et al., "Human mesenchymal stromal cell-derived extracellular vesicles alleviate renal ischemic reperfusion injury and enhance angiogenesis in rats," American Journal of Translational Research, vol. 8, no. 10, pp. 4289-4299, 2016.

[60] L. A. Reis, F. T. Borges, M. J. Simoes, A. A. Borges, R. Sinigaglia-Coimbra, and N. Schor, "Bone marrowderived mesenchymal stem cells repaired but did not prevent gentamicin-induced acute kidney injury through paracrine effects in rats," PLoS One, vol. 7, no. 9, article e44092, 2012.

[61] R. Wang, M. Lin, L. Li et al., "Bone marrow mesenchymal stem cell-derived exosome protects kidney against ischemia reperfusion injury in rats," Zhonghua Yi Xue Za Zhi, vol. 94, no. 42, pp. 3298-3303, 2014.

[62] G. Q. Ju, J. Cheng, L. Zhong et al., "Microvesicles derived from human umbilical cord mesenchymal stem cells facilitate tubular epithelial cell dedifferentiation and growth via hepatocyte growth factor induction," PLoS One, vol. 10, no. 3, article e0121534, 2015.

[63] D. Gu, X. Zou, G. Ju, G. Zhang, E. Bao, and Y. Zhu, "Mesenchymal stromal cells derived extracellular vesicles ameliorate acute renal ischemia reperfusion injury by inhibition of mitochondrial fission through miR-30," Stem Cells International, vol. 2016, Article ID 2093940, 12 pages, 2016.

[64] F. Collino, S. Bruno, D. Incarnato et al., "AKI recovery induced by mesenchymal stromal cell-derived extracellular vesicles carrying microRNAs," Journal of the American Society of Nephrology, vol. 26, no. 10, pp. 2349-2360, 2015.

[65] B. Wang, H. Jia, B. Zhang et al., "Pre-incubation with hucMSC-exosomes prevents cisplatin-induced nephrotoxicity by activating autophagy," Stem Cell Research \& Therapy, vol. 8, no. 1, p. 75, 2017.

[66] H. Jia, W. Liu, B. Zhang et al., "HucMSC exosomes-delivered 14-3-3 $\zeta$ enhanced autophagy via modulation of ATG16L in preventing cisplatin-induced acute kidney injury," American Journal of Translational Research, vol. 10, no. 1, pp. 101-113, 2018. 
[67] H. Y. Choi, S. J. Moon, B. B. Ratliff et al., "Microparticles from kidney-derived mesenchymal stem cells act as carriers of proangiogenic signals and contribute to recovery from acute kidney injury," PLoS One, vol. 9, no. 2, article e87853, 2014.

[68] K. C. Lin, H. K. Yip, P. L. Shao et al., "Combination of adipose-derived mesenchymal stem cells (ADMSC) and ADMSC-derived exosomes for protecting kidney from acute ischemia-reperfusion injury," International Journal of Cardiology, vol. 216, pp. 173-185, 2016.

[69] S. L. Lindsay, S. A. Johnstone, J. C. Mountford et al., "Human mesenchymal stem cells isolated from olfactory biopsies but not bone enhance CNS myelination in vitro," Glia, vol. 61, no. 3, pp. 368-382, 2013.

[70] S. L. Lindsay, S. A. Johnstone, M. A. McGrath, D. Mallinson, and S. C. Barnett, "Comparative miRNA-based fingerprinting reveals biological differences in human olfactory mucosaand bone-marrow-derived mesenchymal stromal cells," Stem Cell Reports, vol. 6, no. 5, pp. 729-742, 2016.

[71] F. Collino, S. Bruno, M. C. Deregibus, C. Tetta, and G. Camussi, "MicroRNAs and mesenchymal stem cells," Vitamins and Hormones, vol. 87, pp. 291-320, 2011.

[72] G. L. Lin and K. D. Hankenson, "Integration of BMP, Wnt, and notch signaling pathways in osteoblast differentiation," Journal of Cellular Biochemistry, vol. 112, no. 12, pp. 3491-3501, 2011.

[73] L. Ling, V. Nurcombe, and S. M. Cool, "Wnt signaling controls the fate of mesenchymal stem cells," Gene, vol. 433, no. 1-2, pp. 1-7, 2009.

[74] X. Guo and X. F. Wang, "Signaling cross-talk between TGF-beta/BMP and other pathways," Cell Research, vol. 19, no. 1, pp. 71-88, 2009.

[75] E. M. Heinrich and S. Dimmeler, "MicroRNAs and stem cells: control of pluripotency, reprogramming, and lineage commitment," Circulation Research, vol. 110, no. 7, pp. 1014-1022, 2012.

[76] R. Lazzarini, F. Olivieri, C. Ferretti, M. Mattioli-Belmonte, R. Di Primio, and M. Orciani, "mRNAs and miRNAs profiling of mesenchymal stem cells derived from amniotic fluid and skin: the double face of the coin," Cell and Tissue Research, vol. 355, no. 1, pp. 121-130, 2014.

[77] J. J. Montesinos, E. Flores-Figueroa, S. Castillo-Medina et al., "Human mesenchymal stromal cells from adult and neonatal sources: comparative analysis of their morphology, immunophenotype, differentiation patterns and neural protein expression," Cytotherapy, vol. 11, no. 2, pp. 163-176, 2009.

[78] G. Li, X.-a. Zhang, H. Wang et al., "Comparative proteomic analysis of mesenchymal stem cells derived from human bone marrow, umbilical cord, and placenta: implication in the migration," Proteomics, vol. 9, no. 1, pp. 20-30, 2009.

[79] A. Subramanian, G. Shu-Uin, N. Kae-Siang et al., "Human umbilical cord Wharton's jelly mesenchymal stem cells do not transform to tumor-associated fibroblasts in the presence of breast and ovarian cancer cells unlike bone marrow mesenchymal stem cells," Journal of Cellular Biochemistry, vol. 113, no. 6, pp. 1886-1895, 2012.

[80] T. A. Moseley, M. Zhu, and M. H. Hedrick, “Adipose-derived stem and progenitor cells as fillers in plastic and reconstructive surgery," Plastic and Reconstructive Surgery, vol. 118, Supplement, pp. 121S-128S, 2006.
[81] M. S. Tsai, S. M. Hwang, K. D. Chen et al., "Functional network analysis of the transcriptomes of mesenchymal stem cells derived from amniotic fluid, amniotic membrane, cord blood, and bone marrow," Stem Cells, vol. 25, no. 10, pp. 2511-2523, 2007.

[82] R. A. Pelekanos, J. Li, M. Gongora et al., "Comprehensive transcriptome and immunophenotype analysis of renal and cardiac MSC-like populations supports strong congruence with bone marrow MSC despite maintenance of distinct identities," Stem Cell Research, vol. 8, no. 1, pp. 58-73, 2012.

[83] C. M. Kolf, E. Cho, and R. S. Tuan, "Mesenchymal stromal cells. Biology of adult mesenchymal stem cells: regulation of niche, self-renewal and differentiation," Arthritis Research \& Therapy, vol. 9, no. 1, p. 204, 2007.

[84] N. Z. Kuhn and R. S. Tuan, "Regulation of stemness and stem cell niche of mesenchymal stem cells: implications in tumorigenesis and metastasis," Journal of Cellular Physiology, vol. 222, no. 2, pp. 268-277, 2010.

[85] M. L. Bustos, L. Huleihel, M. G. Kapetanaki et al., “Aging mesenchymal stem cells fail to protect because of impaired migration and antiinflammatory response," American Journal of Respiratory and Critical Care Medicine, vol. 189, no. 7, pp. 787-798, 2014.

[86] J. Fafián-Labora, I. Lesende-Rodriguez, P. Fernández-Pernas et al., "Effect of age on pro-inflammatory miRNAs contained in mesenchymal stem cell-derived extracellular vesicles," Scientific Reports, vol. 7, no. 1, article 43923, 2017.

[87] A. Ahluwalia and A. S. Tarnawski, "Critical role of hypoxia sensor - HIF- $1 \alpha$ in VEGF gene activation. Implications for angiogenesis and tissue injury healing," Current Medicinal Chemistry, vol. 19, no. 1, pp. 90-97, 2012.

[88] S. W. Song, K. E. Kim, J. W. Choi et al., "Proteomic analysis and identification of paracrine factors in mesenchymal stem cell-conditioned media under hypoxia," Cellular Physiology and Biochemistry, vol. 40, no. 1-2, pp. 400-410, 2016.

[89] L. Song, Y. J. Yang, Q. T. Dong et al., “Atorvastatin enhance efficacy of mesenchymal stem cells treatment for swine myocardial infarction via activation of nitric oxide synthase," PLoS One, vol. 8, no. 5, article e65702, 2013.

[90] D. J. Ceradini, A. R. Kulkarni, M. J. Callaghan et al., "Progenitor cell trafficking is regulated by hypoxic gradients through HIF-1 induction of SDF-1," Nature Medicine, vol. 10, no. 8, pp. 858-864, 2004.

[91] P. R. Crisostomo, Y. Wang, T. A. Markel, M. Wang, T. Lahm, and D. R. Meldrum, "Human mesenchymal stem cells stimulated by TNF- $\alpha$, LPS, or hypoxia produce growth factors by an NF $\kappa$ B- but not JNK-dependent mechanism," American Journal of Physiology. Cell Physiology, vol. 294, no. 3, pp. C675-C682, 2008.

[92] I. Berniakovich and M. Giorgio, "Low oxygen tension maintains multipotency, whereas normoxia increases differentiation of mouse bone marrow stromal cells," International Journal of Molecular Sciences, vol. 14, no. 1, pp. 2119-2134, 2013.

[93] D. H. Munn and A. L. Mellor, "Indoleamine 2,3 dioxygenase and metabolic control of immune responses," Trends in Immunology, vol. 34, no. 3, pp. 137-143, 2013.

[94] J. Croitoru-Lamoury, F. M. J. Lamoury, M. Caristo et al., "Interferon- $\gamma$ regulates the proliferation and differentiation of mesenchymal stem cells via activation of indoleamine 2,3 dioxygenase (IDO)," PLoS One, vol. 6, no. 2, article e14698, 2011. 
[95] C. Noone, A. Kihm, K. English, S. O'Dea, and B. P. Mahon, "IFN- $\gamma$ stimulated human umbilical-tissue-derived cells potently suppress NK activation and resist NK-mediated cytotoxicity in vitro," Stem Cells and Development, vol. 22, no. 22, pp. 3003-3014, 2013.

[96] F. Gieseke, A. Kruchen, N. Tzaribachev, F. Bentzien, M. Dominici, and I. Muller, "Proinflammatory stimuli induce galectin-9 in human mesenchymal stromal cells to suppress T-cell proliferation," European Journal of Immunology, vol. 43, no. 10, pp. 2741-2749, 2013.

[97] Z. F. Lu, G. C. Wang, C. R. Dunstan et al., "Activation and promotion of adipose stem cells by tumour necrosis factoralpha preconditioning for bone regeneration," Journal of Cellular Physiology, vol. 228, no. 8, pp. 1737-1744, 2013.

[98] J. Xing, T. Hou, H. Jin et al., "Inflammatory microenvironment changes the secretory profile of mesenchymal stem cells to recruit mesenchymal stem cells," Cellular Physiology and Biochemistry, vol. 33, no. 4, pp. 905-919, 2014.

[99] D. Wang, J. S. Park, J. S. F. Chu et al., "Proteomic profiling of bone marrow mesenchymal stem cells upon transforming growth factor betal stimulation," The Journal of Biological Chemistry, vol. 279, no. 42, pp. 43725-43734, 2004.

[100] T. J. Bartosh, J. H. Ylostalo, A. Mohammadipoor et al., "Aggregation of human mesenchymal stromal cells (MSCs) into 3D spheroids enhances their antiinflammatory properties," Proceedings of the National Academy of Sciences of the United States of America, vol. 107, no. 31, pp. 13724-13729, 2010.

[101] T. J. Bartosh, J. H. Ylostalo, N. Bazhanov, J. Kuhlman, and D. J. Prockop, "Dynamic compaction of human mesenchymal stem/precursor cells into spheres self-activates caspasedependent IL1 signaling to enhance secretion of modulators of inflammation and immunity (PGE2, TSG6, and STC1)," Stem Cells, vol. 31, no. 11, pp. 2443-2456, 2013.

[102] J. E. Frith, B. Thomson, and P. G. Genever, "Dynamic threedimensional culture methods enhance mesenchymal stem cell properties and increase therapeutic potential," Tissue Engineering. Part C, Methods, vol. 16, no. 4, pp. 735-749, 2010.

[103] J. K. Carrow, L. M. Cross, R. W. Reese et al., "Widespread changes in transcriptome profile of human mesenchymal stem cells induced by two-dimensional nanosilicates," Proceedings of the National Academy of Sciences of the United States of America, vol. 115, no. 17, pp. E3905-E3913, 2018. 


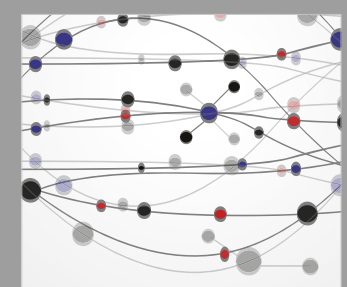

The Scientific World Journal
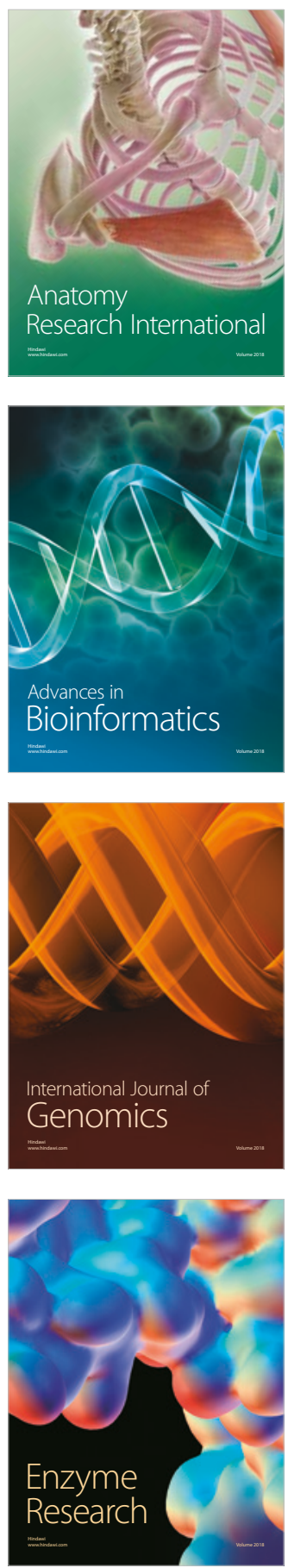
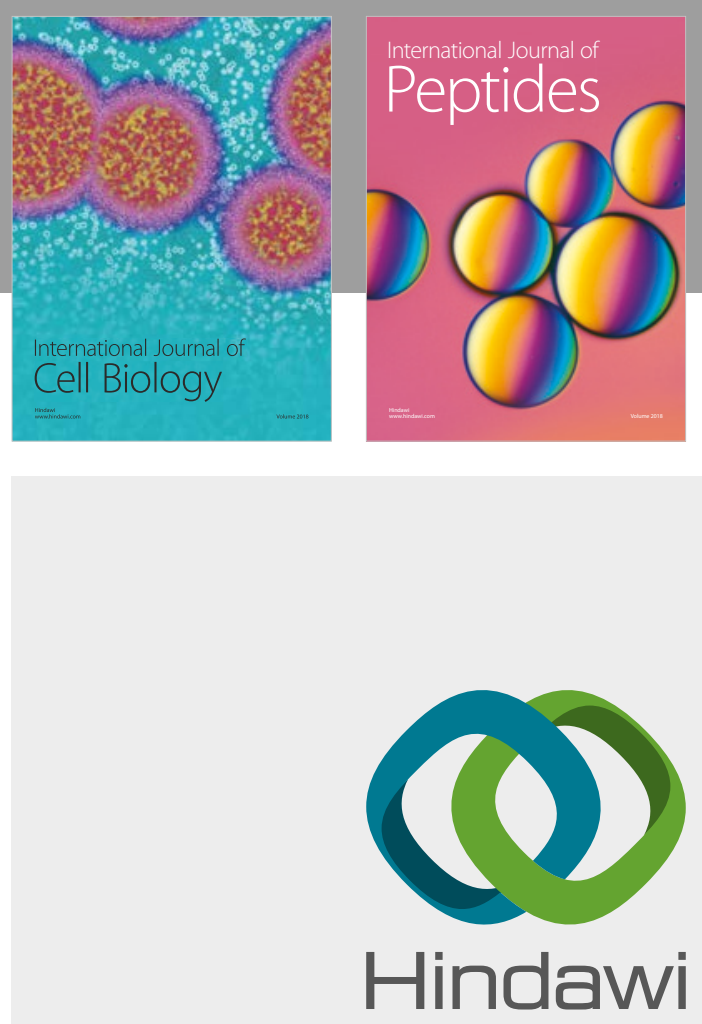

Submit your manuscripts at

www.hindawi.com
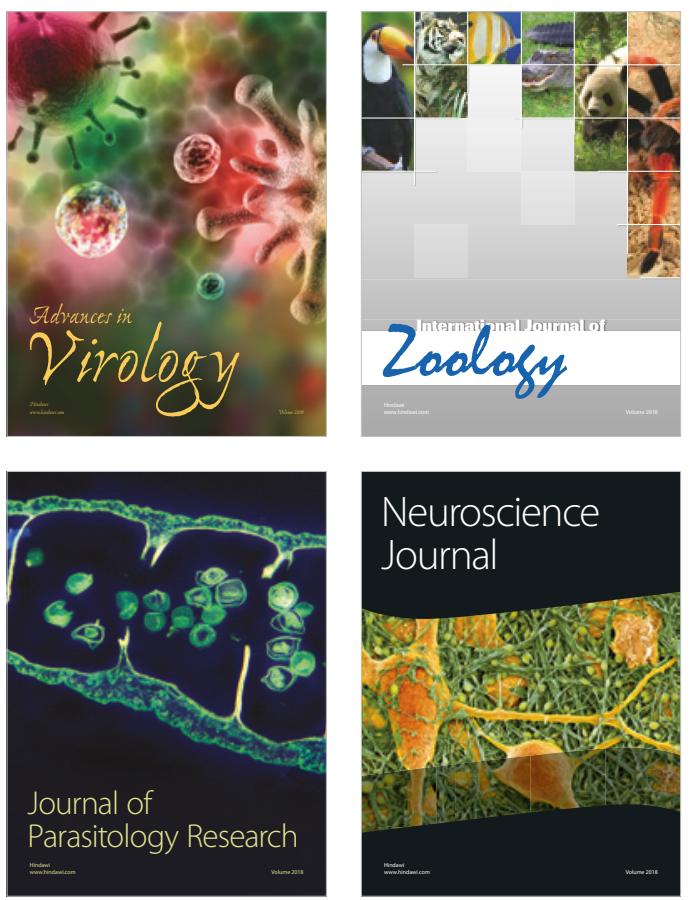
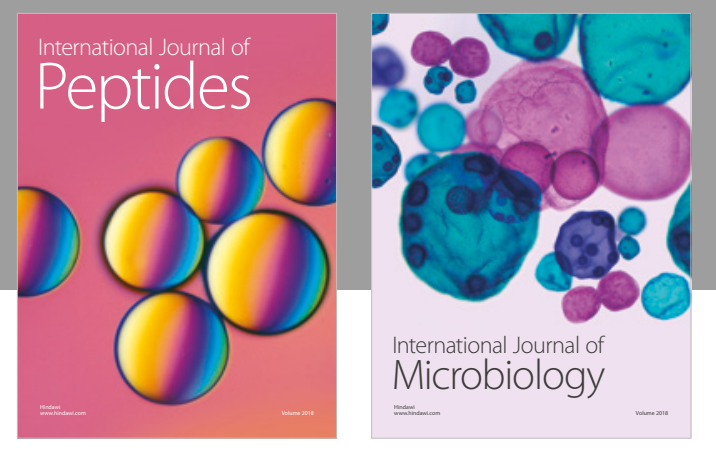

nternational Journal of Microbiology
Journal of
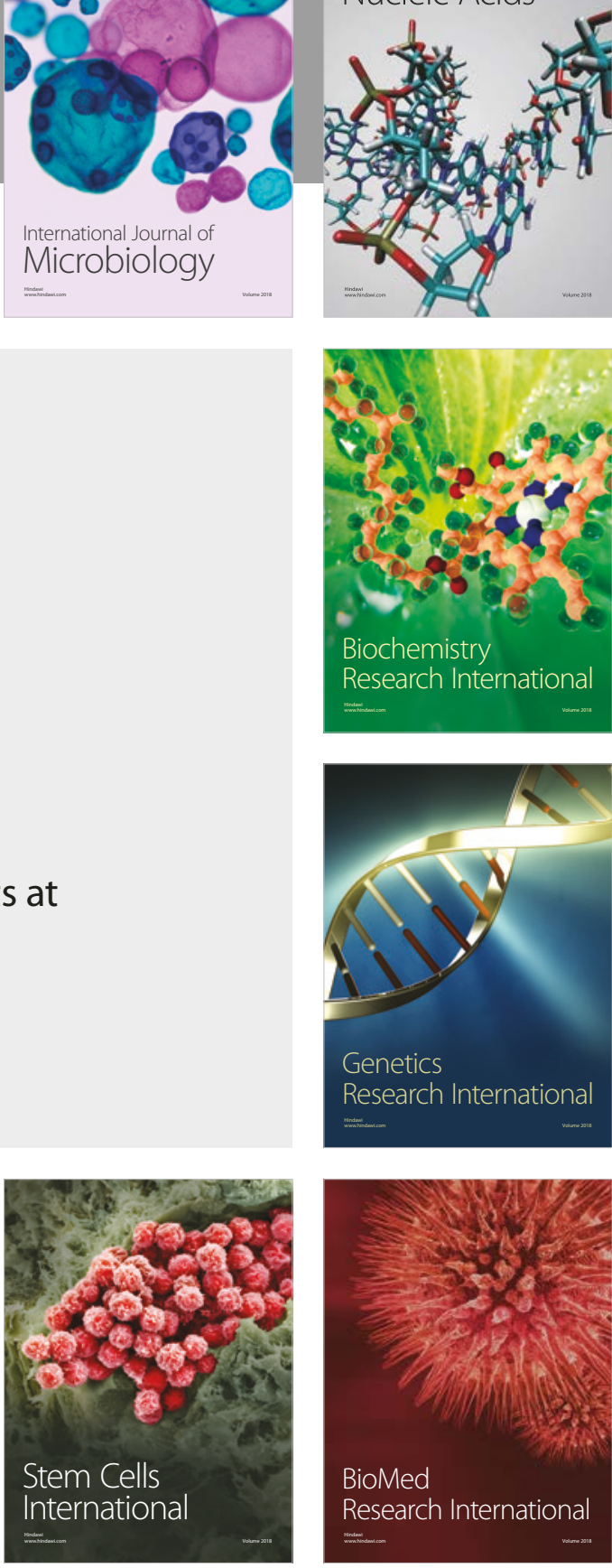
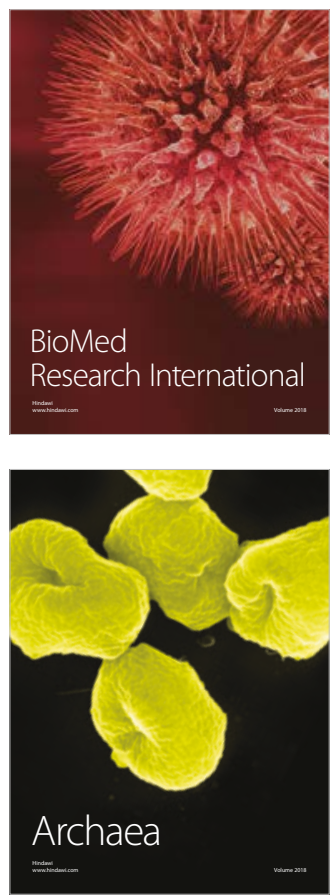